\title{
Elastic properties of mono- and polydisperse two-dimensional crystals of hard-core repulsive Yukawa particles
}

\author{
Jakub W. Narojczyk ${ }^{1}$, Paweł M. Pigłowski ${ }^{1}$, Krzysztof W. Wojciechowski ${ }^{2}$ and \\ Konstantin V. Tretiakov*1 \\ ${ }^{1}$ Institute of Molecular Physics, Polish Academy of Sciences, Smoluchowskiego 17, 60-179 \\ Poznań, Poland \\ ${ }^{2}$ President St. Wojciechowski PWSZ in Kalisz, Nowy Swiat 4, 62-800 Kalisz, Poland
}

July 9, 2021

\begin{abstract}
Monte Carlo simulations of mono- and polydisperse two-dimensional crystals are reported. The particles in the studied system, interacting through hard-core repulsive Yukawa potential, form a solid phase of hexagonal lattice. The elastic properties of crystalline Yukawa systems are determined in the $N p T$ ensemble with variable shape of the periodic box. Effects of the Debye screening length $\left(\kappa^{-1}\right)$, contact value of the potential $(\epsilon)$, and the size polydispersity of particles on elastic properties of the system are studied. The simulations show that the polydispersity of particles strongly influences the elastic properties of the studied system, especially on the shear modulus. It is also found that the elastic moduli increase with density and their growth rate depends on the screening length. Shorter screening length leads to faster increase of elastic moduli with density and decrease of the Poisson's ratio. In contrast to its three-dimensional version, the studied system is non-auxetic, i.e. shows positive Poisson's ratio.
\end{abstract}

\section{Introduction}

Colloidal suspensions are systems consisting of small particles of sizes from tens to hundreds nanometers dispersed in a solvent [1]. The particles can form colloidal crystals not only in three dimensions (3D) [2] but also in two dimensions (2D) where they create a hexagonal lattice [3]. When particles are endowed with electrical charge and placed in a dispersing medium, they exhibit exponential decay of electric potential due to screening effect from ions in the solvent [4]. This decay is described by the screening length $\kappa^{-1}$ (known also as the Debye length) which depends on temperature, permittivity, and concentration of ions in the solvent. Interactions between particles in such systems are decribed by Hard-Core Repulsive Yukawa Potential (HCRYP) [5, 6], both in two [7] and three [5, 8] dimensions. The potential consists of two parts: the hard core (reflecting the influence of finite sizes of particles) and the repulsive part describing spherically symmetric Coulomb screening effect. HCRYP systems can behave (depending on $\kappa^{-1}$ ) as hard particle systems (perfect screening) or as Wigner crystal (no screening) [9].

More than ten years ago the liquid-solid phase diagrams for two-dimensional (2D) [7] and threedimensional (3D) [4, 5, 10, 11] HCRYP systems have been studied. The research showed that in

*kvt@ifmpan.poznan.pl 

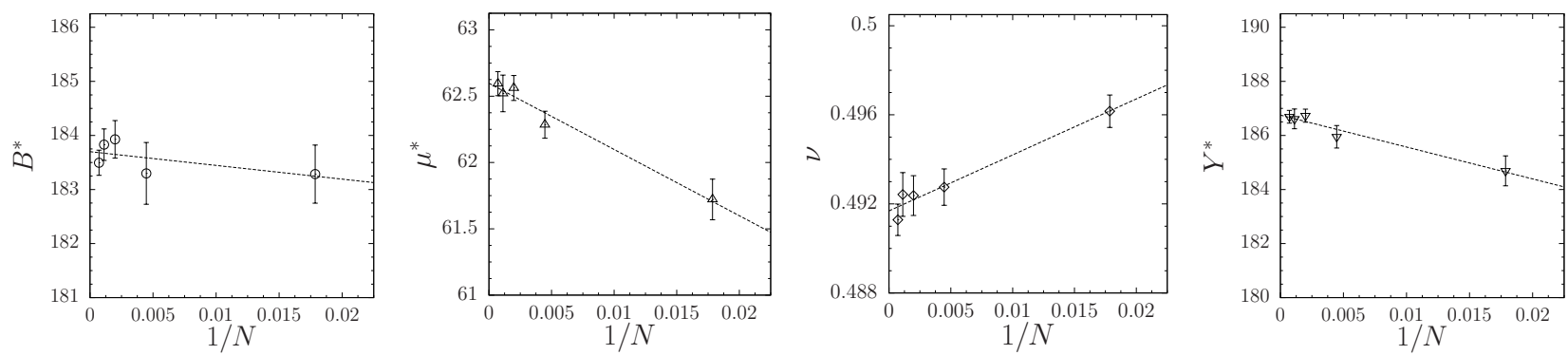

Figure 1: Size dependence of the elastic moduli $B$ (a), $\mu$ (b), Poisson's ratio (c), and Young's modulus (d) for the system consisting of $N=56,224,504,896,1400$ particles, at $p^{*}=30, \beta \epsilon=20$ and $\kappa \sigma=10$. The lines represent linear fits to the obtained data.

2D HCRYP particles can crystallize into a hexagonal lattice, whereas the in 3D they form f.c.c. or b.c.c. structures (depending on external conditions). Various thermodynamic properties and physical phenomena have been studied for the Yukawa potential, both in 2D [7, 9, 12 17] and 3D [46, 10, 11, 18 27]. Recently the elastic properties of three dimensional monodisperse Yukawa system were studied and it was shown that the system is partially auxetic [25].

The term auxetic [28] refers to the class of materials and models that exhibit unusual elastic properties. They expand (shrink) transversally when stretched (compressed) longitudinally. Their Poisson's ratio [29] (in contrast to common materials) is negative. Due to their exceptional properties, auxetic materials and models gather ever growing interest in recent years [30].

It is worth noting that usually colloidal systems are not composed of identical particles. Thus it is meaningful to consider the enhancement of the model by introduction of the particle size dispersion [21, 22]. The study shows that the dispersion in sizes of particles can significantly impact the thermodynamic [31, 32] (especially elastic [33]) properties of the system. The study of polydisperse hard disks system not only indicated a strong dependence of elastic constants on particles size polydispersity, but also revealed surprising behaviour of the Poisson's ratio in the close-packing limit [34]. In this context, the subject of this work are both mono- and polydisperse systems.

This work encompasses the results of study performed on two dimensional hexagonal systems composed of Yukawa particles. The elastic properties of mono- and polydisperse two-dimensional crystals of hard-core repulsive Yukawa particles are described. Beside studies of the influence of screening length, the temperature and the particles size polydispersity on elastic properties, the authors posed additional question: whether this system will also exhibit auxetic properties. It is worth to add that the elastic properties of such a system have never been investigated.

The structure of this manuscript is the following. In section 2 basic formulas and definitions of physical quantities necessary to describe the elasticity of the studied system, as well as the description of models and methods are provided. In section 3 the simulation results and their discussion are presented. The last section (4) provides the summary of the work.

\section{Preliminaries}

\subsection{Elastic properties}

of the system under non-zero external pressure $p$ which is subjected to reversible deformation can be described by expanding the free enthalpy (Gibbs free energy) with respect to the Lagrange strain tensor components. The change of the Gibbs free energy corresponding the elastic deformation of hexagonal lattice under external pressure $p$ expressed with respect to the macroscopic elastic moduli, 

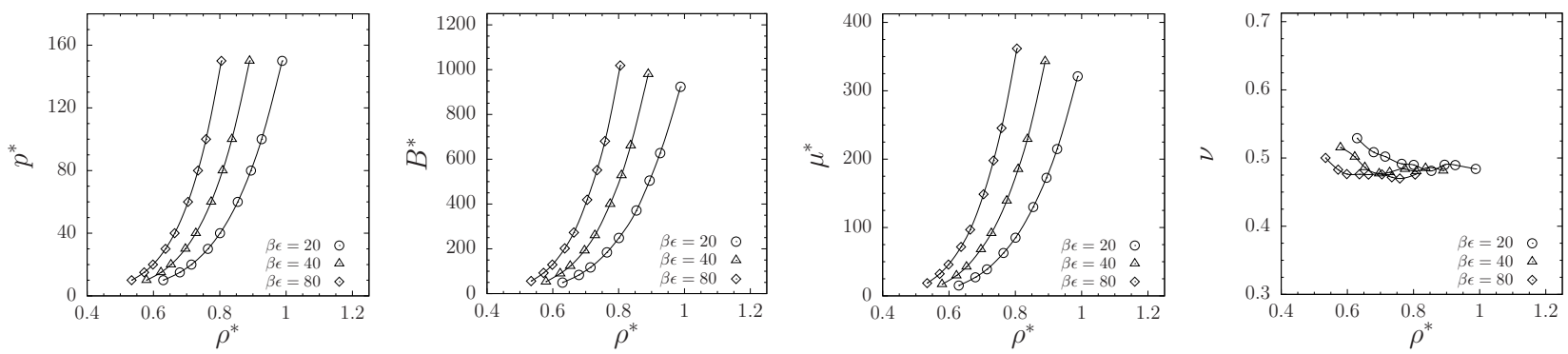

Figure 2: Thermodynamic characteristics of the monodisprese hard-core repulsive Yukawa system at $\kappa \sigma=10$. The pressure $p^{*}(\mathrm{a})$; bulk modulus $B^{*}(\mathrm{~b})$; shear modulus $\mu^{*}(\mathrm{c})$ and the Poisson's ratio $\nu(\mathrm{d})$ are shown as functions of density for three selected values of temperatures. The lines are drawn to guide the eye.
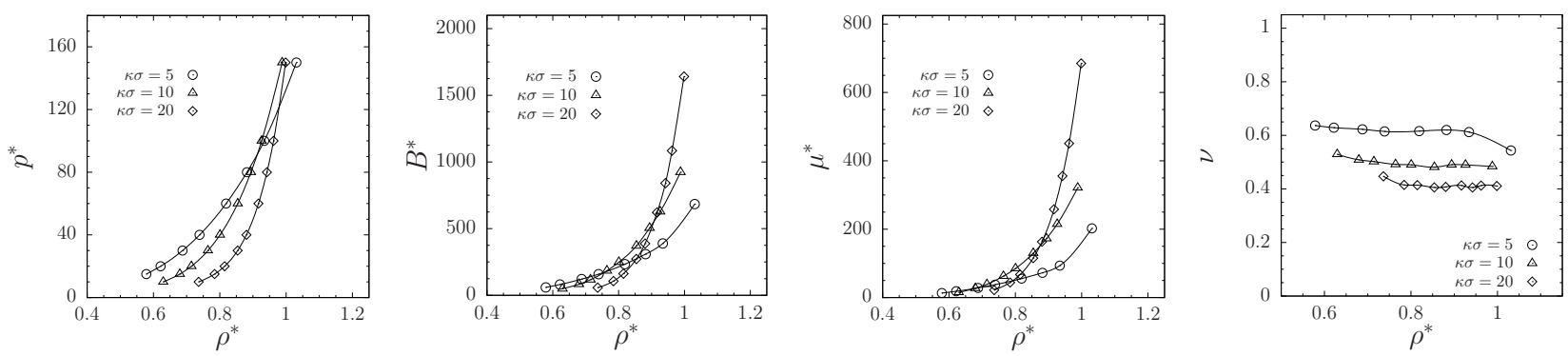

Figure 3: Thermodynamic characteristic of the monodisperse hard-core repulsive Yukawa system at $\beta \epsilon=20$ as functions of density: (a) pressure $p^{*}$, (b) the bulk modulus $B^{*}$, (c) the shear modulus $\mu^{*}$, and (d) the Poisson's ratio. The lines are drawn to guide the eye.

is of the form:

$$
\begin{aligned}
\Delta G / V_{p} & =\frac{1}{2} B\left(\varepsilon_{x x}+\varepsilon_{y y}\right)^{2} \\
& +\frac{1}{2} \mu\left[4 \varepsilon_{x y}^{2}+\left(\varepsilon_{x x}-\varepsilon_{y y}\right)^{2}\right],
\end{aligned}
$$

where $\varepsilon$ is the strain tensor; $V_{p}$ is the reference volume (at equilibrium) of the system under pressure $p ; B$ is the bulk modulus, and $\mu$ is the shear modulus [35]. It follows from eq. (11) that hexagonal lattice is elastically isotropic [29].

\subsection{The Poisson's ratio}

is the negative ratio of the relative changes of transverse to longitudinal dimensions when only the longitudinal stress tensor component is infinitesimally changed. For hexagonal structure in two dimensions under pressure $p$ one can express this quantity in the form [35]:

$$
\nu=\frac{B-\mu}{B+\mu} .
$$

It is worth adding that for $2 \mathrm{D}$ systems the Poisson's ratio can change in the range of $-1 \leq \nu \leq 1$ [35].

\subsection{Particle size dispersion}

was introduced randomly according to the normal distribution with the first two central moments equal to $\sigma$ and $\delta$, respectively. The standard deviation of the particle size distribution divided by 
the mean diameter of the particle, called the polydispersity parameter [32]:

$$
\delta=\frac{1}{\langle\sigma\rangle} \sqrt{\left\langle\sigma^{2}\right\rangle-\langle\sigma\rangle^{2}}
$$

was used to control the degree of disorder in the system. It should be noted that, since the system consists of finite number of particles, it is important to control the values of $\langle\sigma\rangle$ and $\delta$ as well as possible. The simplest way to meet this condition is to repeatedly generate different sets of random numbers until the one is found whose properties fall, within a specified accuracy, near the desired values [36]. In this work, however, a different approach was used. After generating a set of $n$ Gaussian pseudo random numbers (using method described in [37]), the corresponding average $\bar{\sigma}^{(n)}$ and standard deviation $\delta^{(n)}$ are used to convert this set to the standard normal distribution $Z=\left(X-\bar{\sigma}^{(n)}\right) / \delta^{(n)}$. Next one can convert obtained values such that they exactly match any desired distribution $X^{\prime}=(Z-\sigma) / \delta$, with the machine accuracy. This approach ensures the compatibility of distributions for different samples with very high accuracy.

\subsection{The model description}

starts with introducing reduced thermodynamic quantities used in the simulations:

\begin{tabular}{lll}
\hline \hline reduced value & symbol & therm. equiv. \\
\hline inverse temperature & $\beta \epsilon$ & $\epsilon / k_{B} T$ \\
number density & $\rho^{*}$ & $\rho \sigma^{2}$ \\
pressure & $p^{*}$ & $\beta P \sigma^{2}$ \\
bulk modulus & $B^{*}$ & $B \sigma^{2} / k_{B} T$ \\
shear modulus & $\mu^{*}$ & $\mu \sigma^{2} / k_{B} T$ \\
\hline
\end{tabular}

where $T$ is the temperature and $k_{B}$ is the Boltzman constant. The studied system consists of $N$ particles interacting via HCRYP. For the monodispersed systems, the potential of interaction is of the form:

$$
\beta u(r)= \begin{cases}\infty, & r<\sigma, \\ \beta \epsilon \frac{\exp [-\kappa(r-\sigma)]}{r / \sigma}, & r \geq \sigma,\end{cases}
$$

where $r$ is the distance between the centers of particles, $\sigma$ is the diameter of the hard core, $\epsilon$ is the value of pair interaction of particles at contact (i.e. at $r=\sigma$ ) and $\kappa^{-1}$ is the Debye screening length. In the case of systems with non-zero size polydispersity of particles a generalization of the equation (4) proposed by Colombo and Dijkstra [22] was used:

$$
\beta u(r)= \begin{cases}\infty, & r<\bar{\sigma}_{i j}, \\ \beta \epsilon \frac{\sigma_{i} \sigma_{j}}{\bar{\sigma} r} \exp \left[-\kappa\left(r-\bar{\sigma}_{i j}\right)\right], & r \geq \bar{\sigma}_{i j},\end{cases}
$$

\subsection{Computer simulations}

have been performed in the isothermal-isobaric ensemble $(N p T)$ by Monte Carlo method. In order to calculate the elastic moduli, the approach based on the averaging of strain fluctuations proposed by Parrinello-Rahman [38] and further developed by Ray and Rahman [39, 40], was used. The details of the method were described in ref. [41]. It is worth noting that in the case of a potential with long-range interactions the minimum image method (MIM) [42] can be used. Our studies showed that to obtain accurate results for following parameters $\left(\kappa \leq 4, \beta \geq 80, r_{\text {cut }} \leq 2.5\right)$ of studied potential the MIM or another method, which takes into account the long-range interactions, must be used. 
The typical studied systems were composed of $N=224$ particles under periodic boundary conditions. Samples for simulation were chosen such that the edge ratio was as close to 1 as possible. The particles were placed in the $14 a \times 8 \sqrt{3} a$ box, where $a$ is the distance between the nearest neighbors in crystal. The term nearest neighbor refers to particles that share a common edge of their Dirichlet polygons. Results from simulations were averaged over $10^{7}$ Monte Carlo cycles after prior equilibration of $10^{6}$ cycles. To check the dependence of the elastic moduli on the size of studied systems, the system of $N=56,224,504,896,1400$ particles have been studied, for which $5 \cdot 10^{6}, 10^{7}, 3 \cdot 10^{7}, 6 \cdot 10^{7}, 10^{8} \mathrm{MC}$ cycles were performed. The interaction potential implemented for simulations of polydisperse systems is described by the equation (5). For monodisperse systems the latter reduces to the form of eq. (44). Elastic moduli for each phase point were determined by averaging over 10 independent simulations, each of which was performed for a different structure generated from a proper distribution.

\section{Results and discussion}
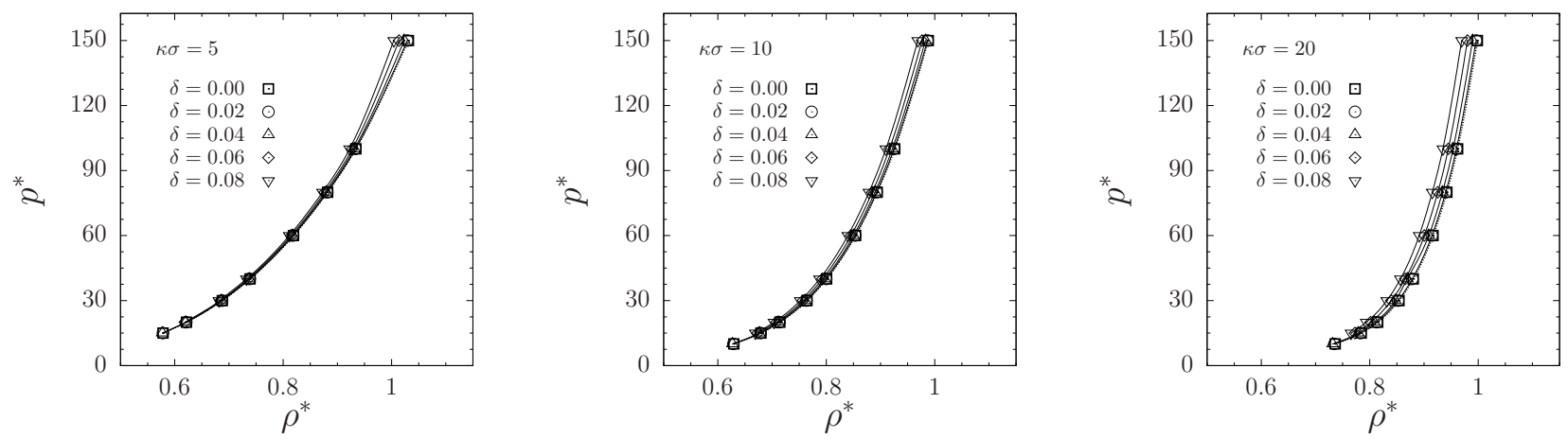

Figure 4: Equation of state of the hard-core repulsive Yukawa system at $\beta \epsilon=20$ for $\kappa \sigma=5$ (a), $\kappa \sigma=10(\mathrm{~b})$, and $\kappa \sigma=20(\mathrm{c})$. The lines are drawn to guide the eye.
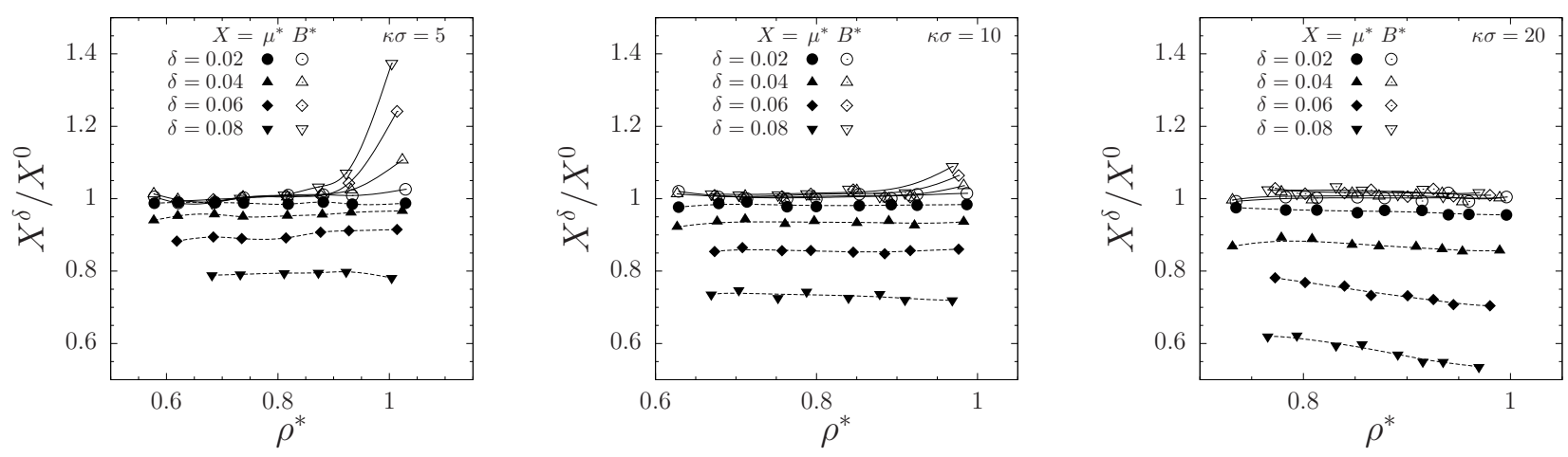

Figure 5: Relative change of elastic moduli due to the non-zero size polydispersity for $\kappa \sigma=5$ (a), $\kappa \sigma=10$ (b) and $\kappa \sigma=20$ (c). $X$ stands for $B$ or $\mu$. The lines are drawn to guide the eye.

Based on the equation of state in Ref. [7], values of the potential's parameters corresponding to solid phases were chosen to allow for comparison of simulation results with experimental data, e.g. $\beta \varepsilon \approx 20$ corresponds to colloidal systems in a low dielectric solvent at room temperature [22]. The discussion of elastic properties of given systems that follows, is presented in the form of the three 

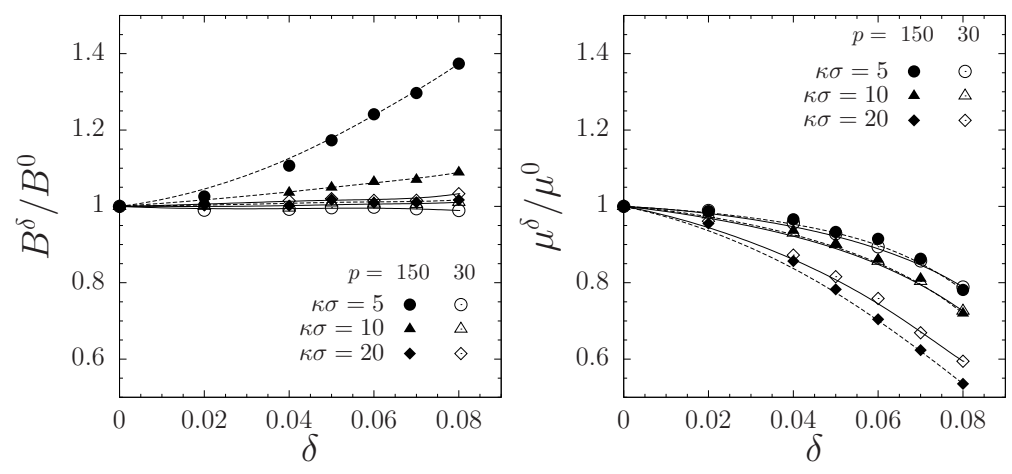

Figure 6: Relative change of (a) the bulk modulus and (b) the shear modulus due to the non-zero size polydispersity, studied at $\beta \epsilon=20$, for two different values of pressure. The lines are drawn to guide the eye.

cases: the constant screening length $(\kappa \sigma)^{-1}$ (sec. 3.1); the constant temperature $(\beta \epsilon)^{-1}$ (sec. 3.2); and the influence of particle size polydispersity (sec. 3.3) on the mentioned above properties.

In Figure 1 are shown dependencies of the elastic moduli and Poisson's ratio with respect to the size of the system. It may be noted that the simulations performed for $N=224$ yield results that differ only a few percent from the values extrapolated to the thermodynamic limit $(N \rightarrow \infty)$. One can add that the agreement is quite good for all the studied system sizes. In view of the above, all further discussion applies to systems containing $N=224$ particles.

\subsection{The case of constant screening length}

is illustrated in Figure 2, The elastic moduli as functions of density for $(\kappa \sigma)^{-1}=10^{-1}$ are presented there. One can observe a monotonic increase of both elastic moduli and pressure with increasing density. A decrease of the temperature results in an increase of pressure and elastic moduli at entire range of studied densities. Furthermore, it causes a shift of the entire isotherm describing the lower temperature in the direction of high densities.

The density dependence of Poisson's ratio is presented in Fig. 2 $\mathrm{d}$. It is immediately apparent that for selected $\kappa$ values of Poisson's ratio for different temperatures are very close one to another at high densities. This suggests that the influence of particles' hard cores becomes dominant at these densities. At low densities an increase of temperature causes an increase of the Poisson's ratio, however the dependence on temperature is rather weak.

\subsection{The case of constant temperature}

was investigated for $(\beta \epsilon)^{-1}=20^{-1}$. Figure 3 illustrates the density dependence of pressure (a) as well as the bulk modulus (b), the shear modulus (c) and the Poisson's ratio (d). Similarly to previous case, an increase of the pressure and the elastic moduli with the density can be observed. However, the growth rate of the elastic moduli is different and it clearly depends on the screening length. An increase of the elastic moduli with increasing density is slower for long-range potential $\left((\kappa \sigma)^{-1}=5^{-1}\right)$ than for interactions with shorter screening length $\left((\kappa \sigma)^{-1}=20^{-1}\right)$.

Figure 3 $\mathrm{d}$ illustrates the dependence of Poisson's ratio on density for systems with different screening lengths. It is noticeable that for a given $\kappa$ the Poisson's ratio weakly depends on the density of the system. However, the screening length itself essentially influences the value of Poisson's ratio. This leads to conclusion that one can control the Poisson's ratio by modifying the Debye screening 

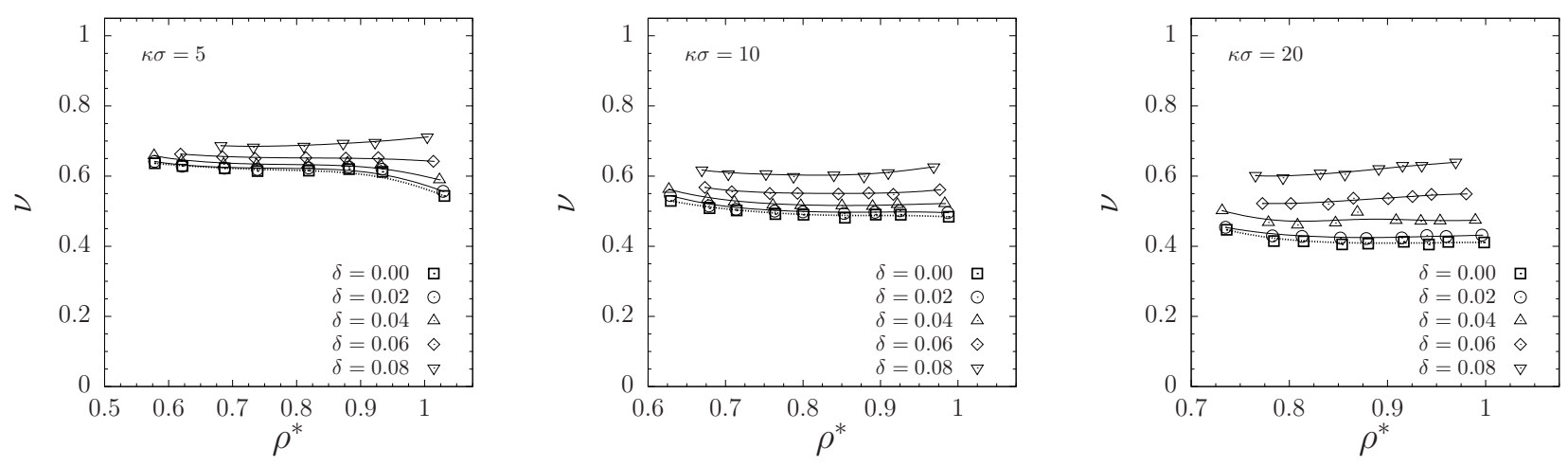

Figure 7: Density dependence of the Poisson's ratio of the polydisperse hard-core repulsive Yukawa system at $\beta \epsilon=20$, for three values of the screening length $\kappa \sigma=5$ (a), $\kappa \sigma=10$ (b), and $\kappa \sigma=20$ (c).

length (e.g. by changing the properties of the electrolyte).

\subsection{Influence of size polydispersity}

of the particles' hard cores on elastic properties is discussed in this section. One can start from the examination of the equation of state presented in the Figure 4. As in the previous case, presented data correspond to systems at reduced temperature $(\beta \epsilon)^{-1}=20^{-1}$ for three values of screening length $(\kappa \sigma)^{-1}: 5^{-1}, 10^{-1}$, and $20^{-1}$. The choice of these values is dictated by two factors. Firstly, studies of Poisson's ratio in case of monodisperse system (see sec. 3.1) showed that its temperature dependence is rather week while the screening length has a significant impact on its value. Secondly, the present study is mainly focused on looking for mechanisms affecting Poisson's ratio. Thus the influence of the screening length on the elastic properties and Poisson's ratio in systems with non-zero polydispersity of particles sizes was considered at a selected (constant) temperature. Systems with four different values of polydispersity parameter $\delta$ have been investigated. Its influence manifests as the change of number density (i.e. the ratio of the number of particles per $2 \mathrm{D}$ 'volume') for a given pressure (see Fig. 4). An increase of the size polydispersity causes a decrease of the number density at a given pressure. The decrease, however, depends on the screening length. For long-range interactions (Fig. 4h) the impact of size dispersion is significantly smaller and noticeable only at high pressures. Once the screening length is getting smaller (increasing $\kappa$ ), rising $\delta$ enforces the system to expand even at lower pressures.

Figure 5 illustrates changes of elastic moduli caused by the size polydispersity relative to monodisperse system for different screening lengths. The relations are presented as functions of density. One can notice that the influence of the size polydispersity is very different for both the moduli. In the case of the bulk modulus, a significant increase can be observed only for high densities and long interactions (Fig $5 \mathrm{~h}$ ). For lower densities the influence of the size polydispersity on the bulk modulus is negligible. Furthermore, when the effect of particles' hard cores becomes dominant (increasing $\kappa)$, one can observe only minor impact of the non-zero polydispersity on $B$ in the whole range of studied densities (Fig. F ). This effect is further illustrated in the Figure 6 a, where the relative bulk modulus with respect the polydispersity parameter is presented for three screening lengths at high and low pressures. It can be seen there that only for long-range interactions and high pressure one can observe a significant influence of polydispersity on the bulk modulus, and this impact quickly decays with the decrease of the screening length.

The effect of the size polydispersity on the shear modulus $\mu$ is stronger than on the bulk modulus. In Figure $5 \mathrm{a}$ one can see that an increase of $\delta$ decreases the shear modulus. From the other 
hand shortening of the screening length increases an influence of the polydispersity on the shear modulus (Fig. [5 and [6). Thus, one can conclude that for a system with given size polydispersity of particles one can additionally control its (the system) susceptibility to the shear deformations by changing the Debye screening length (e.g. by modifying the properties of the electrolyte). Unlike the bulk modulus, pressure has a little impact on a decrease of $\mu$ with the increasing parameter of polydispersity (Fig. [6) . Only in the case of shortest screening lengths $(\kappa \sigma=20)$ the difference between the considered properties of systems under low and high pressures is noticeable. For smaller values of $\kappa$ only an increase of the polydispersity parameter is responsible for a decrease of the shear modulus. It is worth noting, that most significant decrease of the shear modulus is observed for the shortest screening length.

Figure 7 shows the density dependence of the Poisson's ratio for different values of polydispersity parameter and various screening lengths. It can be seen that for long-range interactions the size polydispersity of particles affects weakly the Poisson's ratio and it weakly depends on the density. With shortening the screening length, the impact of polydispersity becomes more noticeable, getting very significant role for the short-range interactions.

\section{Summary and conclusions}

The influence of the temperature, screening lengths and size polydispersity on elastic properties of the two-dimensional hexagonal structure of hard-core, repulsive Yukawa particles was investigated by Monte Carlo simulations in the $N p T$ ensemble with variable shape of the periodic box. The obtained results show that influence of the screening length on the elastic properties of the studied systems is significant. It was also shown that size polydispersity impacts the elastic properties, especially the shear modulus, which decreases with decreasing the size polydispersity parameter. However, an increase of particle size dispersion is reflected in the increase of Poisson's ratio. It was shown that the 2D Yukawa model is non-auxetic but the value of the Poisson's ratio decreases with decreasing screening length. This mechanism can be useful in designing systems with low Poisson's ratio.

\section{Acknowledgement}

We are grateful to Dr. Mikołaj Kowalik (Pennsylvania State University) for discussions. This work was supported partially by the Polish National Science Center grants DEC-2012/05/B/ST3/03255. The calculations were partially performed at Poznań Supercomputing and Networking Center (PCSS).

\section{References}

[1] A. P. Gast and W. B. Russel, Phys. Today 51, 24 (1998).

[2] W. B. Russel, D. A. Saville, and W. R. Schowalter, Colloidal Dispersions (Cambridge University Press, Cambridge, 1991).

[3] P. Pieranski, Phys. Rev. Lett. 45, 569 (1980).

[4] S. Auer and D. Frenkel, J. Phys.: Condens. Matter 14, 7667-7680 (2002).

[5] F. E. Azhar, M. Baus, and J. P. Ryckaert, J. Chem. Phys 112, 5121-5126 (2000).

[6] A. P. Hynninen and M. Dijkstra, J. Phys.: Condens. Matter 15, 3557-3567 (2003). 
[7] R. Asgari, B. Davoudi, and B. Tanatar, Phys. Rev. E 64, 41406 (2001).

[8] A. P. Hynninen and M. Dijkstra, Phys. Rev. E 68, 021407 (2003).

[9] L. Assoud, R. Messina, and H. Lowen, J. Chem. Phys. 129, 164511 (2008).

[10] B. Davoudi, M. Kohandel, M. Mohammadi, and B. Tanatar, Phys. Rev. E 62, 6977 (2000).

[11] N. M. Dixit and C. F. Zukoski, J. Phys.: Condens. Matter 15, 1531-1552 (2003).

[12] T. Glanz and H. Lowen, J. Phys.: Condens. Matter 24, 464114 (2012).

[13] P. Hartmann, G. J. Kalman, Z. Donko, and K. Kutasi, Phys. Rev. E 72, 26409 (2005).

[14] Y. V. Khrustalyov and O. S. Vaulina, Phys. Rev. E 85, 46405 (2012).

[15] O. S. Vaulina and X. G. Koss, Phys. Lett. A 378, 719-722 (2014).

[16] O. S. Vaulina and X. G. Koss, Phys. Lett. A 378, 3475-3479 (2014).

[17] A. Radzvilavicius, Phys. Rev. E 85, 51111 (2012).

[18] M. Heinen, P. Holmqvist, A. J. Banchio, and G. Nagele, J. Chem. Phys 134, 44532 (2011).

[19] S. Zhou and X. Zhang, J. Phys. Chem. B 107, 5294-5299 (2003).

[20] K. Krerner, M. O. Robbins, and G. S. Grest, Phys. Rev. Lett. 57, 2694-2697 (1986).

[21] M. N. van der Linden, A. van Blaaderen, and M. Dijkstra, J. Chem. Phys. 138, 114903 (2013).

[22] J. Colombo and M. Dijkstra, J. Chem. Phys 134, 154504 (2011).

[23] J. Gapinski, G. Nagele, and A. Patkowski, J. Chem. Phys 136, 24507 (2012).

[24] J. Gapinski, G. Nagele, and A. Patkowski, J. Chem. Phys 141, 124505 (2014).

[25] K. V. Tretiakov and K. W. Wojciechowski, Phys. Status Solidi B 251, 383-387 (2014).

[26] G. J. Pauschenwein and G. Kahl, J. Phys.: Condens. Matter 21, 474202 (2009).

[27] E. J. Meijer and F. E. Azhar, J. Chem. Phys 106, 4678 (2007).

[28] K. E. Evans, Endeavour, New Series 15, 170 (1991).

[29] L. D. Landau and E. M. Lifshits, Theory of Elasticity, 3rd Edition (Pergamon Press, Oxford, 1993).

[30] K. L. Alderson1, A. Alderson, J. N. Grima, and K. W. Wojciechowski, Phys. Status Solidi B 251, 263-266 (2014).

[31] S. E. Phan, W. B. Russel, Z. Cheng, J. Zhu, P. M. Chaikin, J. H. Dunsmuir, and R. H. Ottewill, Phys. Rev. E 54, 6633-6645 (1996).

[32] S. E. Phan, W. B. Russel, J. X. Zhu, and P. M. Chaikin, J. Chem. Phys. 108, 9789 (1998). 
[33] K. W. Wojciechowski and J. Narojczyk, Rev. Adv. Mater. Sci. 12(2), 120-126 (2006).

[34] K. V. Tretiakov and K. W. Wojciechowski, J. Chem. Phys. 136, 204506 (2012).

[35] K. W. Wojciechowski, Phys. Lett. A 137, 60-64 (1989).

[36] K. V. Tretiakov and K. W. Wojciechowski, Phys. Status Solidi B 250(10), 2020-2029 (2013).

[37] M. P. Allen and D. J. Tildesley, Computer Simulation of Liquids (Clarendon Press, Oxford, 1987).

[38] M. Parrinello and A. Rahman, J. Chem. Phys. 76, 2662 (1982).

[39] J. R. Ray and A. Rahman, J. Chem. Phys. 80, 4423 (1984).

[40] J. R. RaY and A. Rahman, J. Chem. Phys. 82, 4243 (1985).

[41] K. W. Wojciechowski, K. V. Tretiakov, and M. Kowalik, Phys. Rev. E 67, 036121 (2003).

[42] K. V. Tretiakov and K. W. Wojciechowski, Comput. Phys. Commun. 189, 77-83 (2015). 